\title{
Surface-Gravity Inequalities and Generic Conditions for Strong Cosmic Censorship
}

\author{
Wenceslao Santiago-Germán \\ Department of Physics, University of Newcastle upon Tyne, NE1 7RU,U.K*
}

(Dated: November 19, 2018)

\begin{abstract}
Transforming Penrose's intuitive picture of a strong cosmic censorship principle - that generically forbids the appearance of locally naked space-time singularities - into a formal mathematical proof, remains at present, one of the most outstanding unsolved mathematical problems from the theory of gravitational collapse. Part of the difficulty lies in the fact that we do not possess yet a clearcut understanding of the hypothesis needed for the establishment of some sort of strong cosmic censorship theorem. What we have is a selected list of solutions, which at first sight seem to go against cosmic censorship, but at the end they fail in some way. However, the space of solutions of Einstein's field equations is vast. In this article, we plan to increase one's intuition by establishing a link between certain inequalities for Cauchy-horizon stability and a set of generic conditions, such as a reasonable equation of state - which determines whether the space-time is asymptotically flat or not, an energy condition, and an hypothesis over the class of metrics on which Einstein's field equations ought to be solved to ensure strong cosmic censorship inside black-holes. With these tools in hand we examine the Cauchy-horizon stability of the theory created by Born and Infeld-whose action principle has been used as a prototype in superstring theory, and the singularity-free Bardeen's black-hole model.
\end{abstract}

PACS numbers: $04.70 . \mathrm{Bw}, 04.20 . \mathrm{Gz}$

Keywords:

\section{Introduction.}

One of the most striking predictions of Einstein's theory of gravity is the occurrence of space-time singularities, i.e. causal geodesic incompleteness, as a generic feature of classical gravitational collapse. This remarkable conclusion is encapsulated by the singularity theorems of Hawking and Penrose, who rely for its proof on techniques of differential topology [1]. In principle, uncontrollable information may enter the space-time from a singularity and spoil one's ability to predict the future. That possibility has motived the following conjecture due to R. Penrose 2]:

"Generically, the classical general theory of relativity would not allow for the creation of locally naked spacetime singularities."

This statement has been referred to as the strong cosmic censorship conjecture and none knows whether it is true or false. According to this viewpoint, the internal structure of a generic black-hole is bounded by a singularity that does not lie into the past of any space-time point.

The proviso of generic is necessary since, for instance, the maximal analytical extension of the Kerr space-time, which describes an spinning black hole, offers a rich set of possibilities for experience including: close-time like curves, causal trajectories skirting the singularity, and new asymptotically flat regions. However, this analytic extension can not survive the propagation of small perturbations. These perturbations are magnified without

*Electronic address: g.w.santiago-german@ncl.ac.uk limit near the Cauchy horizon, which is then transformed into a singular barrier that seals the region where predictability is lost.

There are some mathematical results where one does not let - just by hand - the initial data develop or evolve outside some restriction on the metric, usually an isometry. The most complete study of this kind, up today, is the one by Isenberg and Moncrief on polarized Gowdy metrics 3], which are vacuum (or electrovac) solutions of Einstein's field equations having only two Killing vector fields. Remarkably, the strong cosmic censorship conjecture was verified within this restricted space of solutions. In the Cauchy-horizon stability approach, one must, in contrast, examine every conceivable perturbation to the space-time background which may lead to instability, only restricted by what one should consider physically viable.

Before developing a general treatment that includes all the spinning black-holes, it is natural to look for clues in charge black-hole space-times since they share certain resemblance with the causal structure of a rotating hole.

Let us thus consider more carefully the ReissneerNordström solution. The mathematical analysis on the propagation of small perturbations of compact support in the Reissneer-Nordström background leads to an unbounded flux of radiation at the future-Cauchy horizon. The slowest rate of divergence-which takes the form $e^{\left(\kappa_{-}-\kappa_{+}\right) v}$, where the advanced time, $v$, goes to plus infinity near the Cauchy horizon - is controlled by an inequality between the surface gravities, $\kappa_{-}$and $\kappa_{+}$, at the Cauchy and future event horizons respectively [4].

That is,

$$
-\left(\kappa_{+}-\kappa_{-}\right) \geq 0
$$




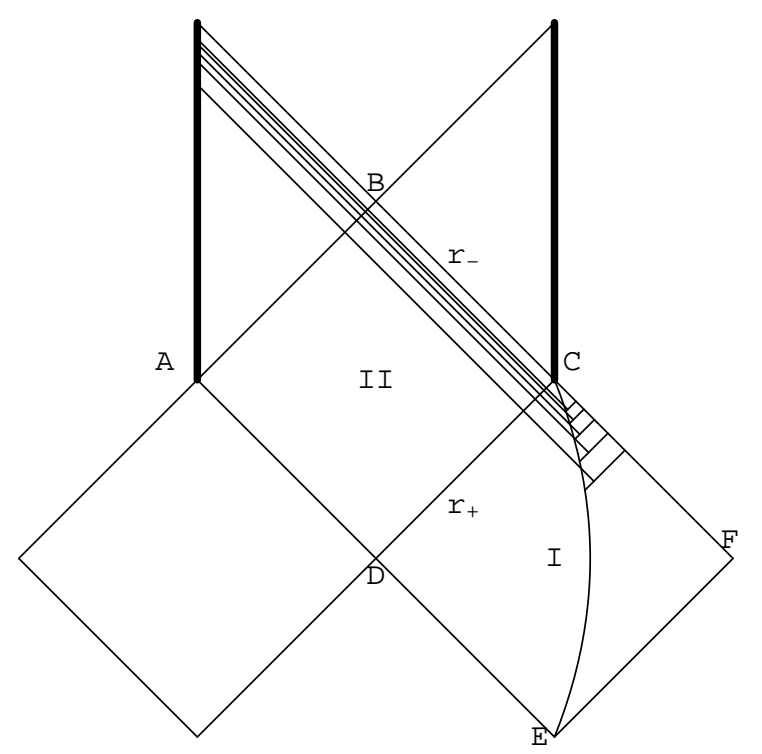

FIG. 1: Penrose diagram for a charge black-hole illustrating how the scattering of perturbations along the arc $\overline{E C}$ will result in an accumulation of wave fronts for the radiation received by an observer crossing the Cauchy horizon along the segment $\overline{B C}$. When the inequality $-\left(\kappa_{+}-\kappa_{-}\right)>0$ between the surface gravities, $\kappa_{-}$and $\kappa_{+}$, of the Cauchy horizon, $\overline{A B C}$, and future event horizon, $\overline{A D C}$, is satisfied, the flux of radiation diverges for all physically reasonable perturbations, even including those with compact support. This assertion still holds in the presence of a positive cosmological when the space-time is asymptotically De Sitter [5]. The $v$-coordinate is defined to be and advanced time which takes the value $+\infty$ at $\overline{B C}$ and $-\infty$ at $\overline{A D}$.

It seems, however, that the role of the surface-gravity inequality (11), is of a more general character beyond the Reissner-Nordström geometry. Its relevance arises because, heuristically, the radiation of compact support is not only blue shifted by approaching the Cauchy horizon but also redshifted by climbing away from the event horizon [5], see FIG 1] Mathematically, the form of this divergence follows from the analytical properties of the black hole scattering potential, or potential well, which vanishes at both horizons.

We argue that this universal inequality, (1), cannot be overcome, precisely, because it turns out to be equivalent - via Einstein's field equations - to a local condition on the source of the gravitational field, which is satisfied by all matter fields obeying some energy condition and a reasonable equation of state (which determines whether the space-time is asymptotically flat or not). Let us illustrate our assertion with an example.

\section{SPHERICAL CONFIGURATIONS OF CHARGE AND MASS}

To simplify things as much as possible, let us consider the metric for a static spherically symmetric gravitational field produced by a spherically symmetric body at rest, i.e.

$$
\begin{aligned}
d s^{2}= & -\left(1-\frac{2 G m(r)}{r}\right) s(r)^{2} d v^{2}+2 s(r) d v d r \\
& +r^{2}\left(d \theta^{2}+\sin ^{2} \theta d \varphi^{2}\right)
\end{aligned}
$$

where $r$ denotes the area radius function. One may think of this metric as roughly describing the asymptotically late-time behavior of a nearly spherical body undergoing gravitational collapse. To simplify further, take a stress-energy tensor satisfying the equation $T_{v}^{v}=T_{r}^{r}$ in Einstein's field equations. The result is that $s(r)$ is now a constant function which can be normalized to plus (or minus) one. For the first option, the area radius function is decreasing towards the future along the ray $v=c t e$.

Theories of non-linear electrodynamics generate solutions of this kind and the black-holes they produce obey the zero and the first law of black-hole mechanics $[\underline{6}$.

In these coordinates, Einstein's law of gravitation leads to (See [7])

$$
\begin{gathered}
\rho=\frac{m_{, r}}{4 \pi r^{2}}, \\
2 p=-\frac{m_{, r r}}{4 \pi r} ;
\end{gathered}
$$

where the mass function, $m(r)$, is given by

$$
\begin{aligned}
\partial^{\mu} r \partial_{\mu} r & =\left(1-\frac{2 G m(r)}{r}\right) \\
& \equiv \frac{\Delta}{r^{2}}
\end{aligned}
$$

and we have introduced the quantity $\Delta$ for notational purposes. The stress-energy tensor results to be diagonal and it has the form $\left(T_{v}^{v}, T_{r}^{r}, T_{\theta}^{\theta}, T_{\phi}^{\phi}\right)=(-\rho,-\rho, p, p)$.

If a static observer has four velocity $\left(U^{v}, U^{r}, U^{\theta}, U^{\phi}\right)=$ $\left(\Delta^{-1 / 2} r, 0,0,0\right)$, he would measure a local energy density, $\rho$, and pressure, $p$, equal to the quantities showed in equations (3) and (4) respectively.

\section{THE ROLE OF ALMOST EXTREME BLACK-HOLES}

Extreme black-holes have zero surface gravity, $\kappa=0$, and in the charge-mass parameter space define a boundary beyond which black-holes do not exist. Therefore, they provide an excellent place to start an analysis about cosmic censorship.

Let us denoted by $\epsilon$ any set of parameters such as the mass, the electric, or magnetic charges of some classical field theory. Although in general, given $\epsilon$, there are no algebraic expressions relating the locations of the horizons of the black-holes generated by these theories with the above physical parameters - for instance, in the BornInfeld theory, one needs to resort to elliptic integrals of 
the first kind - a simple rule applies for near extreme black-holes. A rule, that will allow us to surmount certain technical difficulties and, in fact, avoid the direct calculation of the roots of the equation $\Delta=0$ - which is generic - in order to evaluate the surface gravity inequality (11).

An extreme black-hole satisfy $\Delta=\Delta^{\prime}=0$ at the horizons, where the prime denotes the partial derivative with respect to the area radius function - from there that its surface gravity vanishes since $\kappa=\left|\frac{\Delta^{\prime}}{2 r^{2}}\right|$. Taylor expand $\Delta$ around any of the extreme values in $\mathrm{r}$ and $\epsilon$, say for definiteness $r_{o}$ and $\epsilon_{o}$, then

$$
\begin{aligned}
\Delta(\epsilon, r)= & \Delta_{, \epsilon} \delta \epsilon+\frac{1}{2} \Delta_{, \epsilon \epsilon} \delta \epsilon^{2}+\Delta_{, \epsilon}^{\prime} \delta \epsilon \delta r \\
& +\frac{1}{2} \Delta^{\prime \prime} \delta r^{2}+o\left(\left(\delta r^{2}+\delta \epsilon^{2}\right)^{3 / 2}\right) .
\end{aligned}
$$

At second order, if $\Delta^{\prime \prime} \neq 0$, we end with a quadratic polynomial in $\delta r$. Take $\delta \epsilon$ in the direction that allows the existence of two real roots

$$
\delta r= \pm \frac{1}{\Delta^{\prime \prime}}\left(-2 \Delta^{\prime \prime} \Delta_{, \epsilon} \delta \epsilon\right)^{1 / 2}-\frac{\Delta_{, \epsilon}^{\prime}}{\Delta^{\prime \prime}} \delta \epsilon+o\left(\delta \epsilon^{3 / 2}\right) .
$$

Thus, it is possible to know the positions of the horizons and consequently evaluate the corresponding surface gravities. The result is

$$
\begin{aligned}
\kappa= & \mid \pm \frac{1}{2 r^{2}}\left(-2 \Delta^{\prime \prime} \Delta_{, \epsilon} \delta \epsilon\right)^{1 / 2} \\
& -\frac{\Delta_{, \epsilon}}{2 \Delta^{\prime \prime} r^{3}}\left(r \Delta^{\prime \prime \prime}-4 \Delta^{\prime \prime}\right) \delta \epsilon+o\left(\delta \epsilon^{3 / 2}\right) \mid
\end{aligned}
$$

Here, it is assumed that $g_{\mu \nu} \in C^{3}$. To have a bounded flux of the incident radiation at the future-Cauchy horizon, it is needed that

$$
\kappa_{+}-\kappa_{-}>0
$$

but for nearly extreme black-holes, whose line element is of the form (2), with $s(r)=1$, Eq. (9) implies that (10) is equivalent to

$$
r \Delta^{\prime \prime \prime}-4 \Delta^{\prime \prime}>0,
$$

or more succinctly, in terms of the pressure given by Eq.(4)

$$
\frac{\delta p}{\delta r}>0
$$

which must be evaluated at extreme values.

Expressions (11) or (12) form a simple criterion for examining the disruption of predictability in the interior of nearly extreme black-holes. For instance, it can be seen immediately that despite all the effects produced by the addition of a positive cosmological constant: ray focusing, compact achronal cross sections, etc. It does not help to enforce the Cauchy-horizon-stability requirement (10) since it would give a vanishing contribution to (12)!
This is a consequence of the $C^{3}$-smoothness on the metric tensor [8]. We would like to remark here that in the presence of a positive cosmological constant, say $\Lambda$, besides the stability requirement (10) it is also required to satisfy the inequality $\left(\kappa_{\Lambda}-\kappa_{-}\right)>0$. This extra condition results from the fact of having causally aligned both the cosmological and future black-hole event horizons [5].

Let us now evoke the principle of local conservation of material energy over a small region of the space-time. Taking the $r$-component of $T_{\mu ; \nu}^{\nu}$, we have $\frac{\delta \rho}{\delta r}=-\frac{2(\rho+p)}{r}$. But if $p=p(\rho)$, it follows that

$$
\frac{\delta p}{\delta r}=-\frac{2(\rho+p)}{r} \frac{\delta p}{\delta \rho} .
$$

Hence, by (12) and (13), both the weak energy condition, i.e. $\rho \geq 0$ and $p+\rho \geq 0$, and an equation of state for the source of gravitational field satisfying $\frac{\delta p}{\delta \rho} \geq 0$ imply the validity of the strong cosmic censorship principle inside black-holes closed enough to extremality, that possess a $C^{3}$-metric of the form (2) with $s(r)=c t e$.

\section{BARDEEN BLACK-HOLES}

The Bardeen model is a singularity-free black-hole solution representing the self-gravitating field of a nonlinear magnetic monopole satisfying the weak energy condition [9]. The line-element of this hole has the form (2), with $s=1$, but the mass-function is given by

$$
m(r)=\frac{M r^{3}}{\left(r^{2}+g^{2}\right)^{3 / 2}},
$$

where $g$ denotes the monopole charge and $M$ is the ADM mass. Black-hole space-times occur when the monopole charge is bounded by the ADM mass as follows

$$
\frac{3 \sqrt{3}}{2}|g| \leq 2 M G
$$

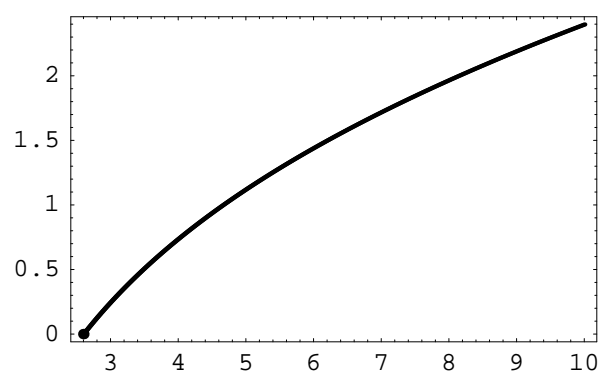

FIG. 2: $-|g|\left(\kappa_{+}-\kappa_{-}\right)$vs. $2 G M /|g|$. This graph shows the Cauchy-horizon instability of Bardeen's black-hole. Near the extreme value $2 M_{o} G /\left|g_{o}\right|=\frac{3 \sqrt{3}}{2}$ (small circle), the instability can be deduced from Eq. (16). Whereas when $M$ goes to infinity, $-|g|\left(\kappa_{+}-\kappa_{-}\right)$approaches to $(2 G M /|g|)^{1 / 2}$ remaining positive. 
The limiting case, when this inequality is saturated, corresponds to an extreme black-hole whose horizon's area is equal to $\mathcal{A}=8 \pi g^{2}$.

The maximal analytic extension of these space-times is free of space-time singularities. However, as can be readily seen by the Cauchy-horizon-stability criteria (12), near the extreme case, the analytic extension is unstable since

$$
\left(\frac{\delta p}{\delta r}\right)_{\left(\epsilon_{0}, r_{o}\right)}=-\frac{5 M}{54 \pi \sqrt{6} g^{4}} \leq 0
$$

This conclusion about instability holds true for the rest of the space of physical parameters, as shown in FIG 2

\section{VARIATION FORMULAS FOR NO EXTREME BLACK-HOLES}

In view of the success of establishing a link between an inequality - which involves two closed but separated events in the space-time - and the local properties of the matter field that generates the near extreme black-hole background; one may ask whether the current local hypothesis cannot be extended to cover all other cases as well, where near extreme conditions do not occur. If cosmic censorship is a principle of classical physics, it should manifest itself as a consequence of the local rules in which the laws of classical physics are formulated. Hence, it seems reasonable to imagine that one may proceed to develop a topological argument, in which the vanishing of the quantity $\kappa_{+}-\kappa_{-}$is forbidden unless one reaches a point representing an extreme black-hole. Then, by continuity, and the results of the last section, the neighborhood where $-\left(\kappa_{+}-\kappa_{-}\right) \geq 0$ should necessarily cover the whole simple-connected space of physical parameters that allows the existence of two horizons. Let us see what are the consequences of adopting this kind of approach.

Firstly, since it is technically difficult to find roots for non-algebraic equations, let us change of variables, from the total charge and ADM mass $\left(q^{2}, M\right)$ to the horizon positions $\left(r_{-}, r_{+}\right)$. Then, by virtue of Einstein's field equations, the determinant of the relevant Jacobian is given by

$$
\operatorname{det} \frac{\partial\left(r_{-}, r_{+}\right)}{\partial\left(q^{2}, M\right)}=\frac{4 \pi G^{2}}{r_{+} r_{-} \kappa_{+} \kappa_{-}} \int_{r_{-}}^{r_{+}}\left(\partial_{, q^{2}} \rho\right) r^{2} d r
$$

This transformation is invertible if the horizons are not degenerate and a change in the total energy density, due to an increase in the charge, $\delta q^{2}$, enclosed between horizons is not zero. Such a description also leads to the variation formula

$$
-\delta\left(\kappa_{+}-\kappa_{-}\right)=-G\left(\frac{\kappa_{+}, r_{+}}{r_{+} \kappa_{+}}+\frac{\kappa_{-, r_{-}}}{r_{-} \kappa_{-}}\right) \delta M+2 \pi\left(w_{+}\left[m_{, q^{2}}, \kappa\right]+w_{-}\left[m_{, q^{2}}, \kappa\right]\right) \delta q^{2}
$$

where we have defined

$$
w[f, g] \equiv f^{\prime} g-g^{\prime} f ; \quad f^{\prime}=\frac{1}{\frac{1}{4 G} \kappa} \partial_{\mathcal{A}} f,
$$

to denote the Wronskian between two functions of the area element, $\mathcal{A}=4 \pi r^{2}$. The next step is to evaluate the function $\chi\left(M, q^{2}\right)=-\left\{\kappa_{+}\left(M, q^{2}\right)-\kappa_{-}\left(M, q^{2}\right)\right\}$ at a critical point. Let us assume that the pressures $p_{+}$and $p_{-}$, at $r_{+}$and $r_{-}$respectively, are positive. Then, the vanishing of the variation of $-\left(\kappa_{+}-\kappa_{-}\right)$with respect to the ADM mass, $M$, implies

$$
\frac{p_{+}}{p_{-}}>\frac{\kappa_{+}}{\kappa_{-}} .
$$

Thus, we have that $-\left(\kappa_{+}-\kappa_{-}\right)>0$ at a critical point of $\chi$ (if this exits) since $p$ is assumed to be $r$-decreasing in order to cover the near extreme case. To prove (1), we shall add a further hypothesis:

$$
\lim _{r \rightarrow 0^{+}} \rho=\frac{a^{2}}{8 \pi G} r^{-\gamma} ; \quad \gamma \geq 2 .
$$

If $\gamma=2$, take $a^{2}>1$. In effect, Eq. 21) implies that $\lim _{r_{-} \rightarrow o^{+}}-\left(\kappa_{+}-\kappa_{-}\right)=+\infty$. Being $\chi\left(M, q^{2}\right)$ a continu- ous function that does not vanish except at an extreme point. We conclude, by varying $M$ and taking different values of $q^{2}$, that the surface-gravity inequality (11) holds. In such circumstances, strong cosmic censorship holds for not necessarily extreme black-holes as well.

\section{THE BORN-INFELD ACTION}

The postulate of the constancy of the velocity of light in relativistic mechanics means that there is an upper limit to the velocity of massive particles moving along a path of maximal length. In 1934, Born and Infeld suggested to put forward the classical foundations of electrodynamics on a similar basis by adding two postulates:

1. The existence of an absolute constant, $1 / b$, placing an upper limit to the magnitude of purely electric fields, and 2. The Lagrangian of the theory to be the square root of a determinant (see Ref.[10]).

The Born-Infeld action then follows from the requirement of having Maxwell theory as the limit when $b$ goes 
to zero. Its Lagrangian density reads

$$
L=\frac{1}{b}\left\{\sqrt{-g}-\sqrt{-\operatorname{det}\left(g_{\mu \nu}+b F_{\mu \nu}\right)}\right\}
$$

or

$$
L=\frac{1}{b}\left\{1-\sqrt{1+b^{2}\left(B^{2}-E^{2}\right)-b^{4}(E \cdot B)^{2}}\right\} .
$$

By Birkhoff's theorem, the spherically symmetric electrovac solution of Einstein's field equations coupled to a Born-Infeld field is static and has the form

$$
d s^{2}=-\left(1-\frac{2 G m}{r}\right) d t^{2}+\left(1-\frac{2 G m}{r}\right)^{-1} d r^{2}+r^{2} d \Omega^{2},
$$

[1], where

$$
\begin{aligned}
m(r)= & \frac{1}{b^{2}} \int_{0}^{r}\left(\sqrt{\left(4 \pi x^{2}\right)^{2}+b^{2}\left(Q^{2}+P^{2}\right)}-4 \pi x^{2}\right) d x \\
& -\left(\frac{\left(Q^{2}+P^{2}\right)^{3 / 4}}{3 \sqrt{\pi b}} \int_{0}^{\infty} \frac{1}{\sqrt{y^{4}+1}}-M\right) .
\end{aligned}
$$

It depends on the electric-magnetic dual invariant combination, $Q^{2}+P^{2}$, that we set equal to $q^{2}$, and the ADM mass, $M$, which appears as an integration constant. Here, the electromagnetic energy and pressure are given by

$$
\rho=-L+E \cdot \frac{\partial L}{\partial E}=\frac{1}{b^{2}}\left(\sqrt{1+\frac{b^{2} q^{2}}{\left(4 \pi r^{2}\right)^{2}}}-1\right)
$$

and

$$
p=L-B \cdot \frac{\partial L}{\partial B}=\frac{1}{b^{2}}\left(1-\frac{1}{\sqrt{1+\frac{b^{2} q^{2}}{\left(4 \pi r^{2}\right)^{2}}}}\right)
$$

respectively. Therefore, its equation of state is

$$
p=\frac{\rho}{1+b^{2} \rho}
$$

and the dominant energy condition, $-\rho \leq p \leq \rho$, holds.

The spherically symmetric stationary black-holes of the Born-Infeld theory, whose pure electric field - we recall - has bounded magnitude, do not provide counterexamples to strong cosmic censorship since:

i) Their energy density and pressures are positive,

ii) Their equation of state, of the form $p=\frac{\rho}{1+b^{2} \rho}$, satisfies $\frac{\delta p}{\delta \rho} \geq 0$

iii) $\lim _{r \rightarrow o^{+}} \rho=\frac{2 G|q| / b}{8 \pi G} r^{-2}$.

The fact that $2 G|q| / b>1$ is a necessary requirement for having two horizons with $r_{+} \neq r_{-}$. Since $\gamma=2$, we see that the Born-Infield theory corresponds to a limiting case of Eq. (21)

\section{GENERALIZATION CAPABILITY}

The criterion expressed by the inequality (see (11))

$$
\left\{-\left(r \Delta^{\prime \prime \prime}-4 \Delta^{\prime \prime}\right)\right\}_{\left(r_{o}, \epsilon_{o}\right)} \geq 0,
$$

which would imply - if verified - the establishment of some sort of strong cosmic censorship principle, can be generalized in a form that does not rely on the spherical assumption, and where the nature of having two non degenerate Killing horizons is seen manifestly.

In effect, for a sufficiently nearly extreme black-hole let us use a chart, covering the region between the two horizons, in a manner of the ADM $3+1$ splitting

$$
d s^{2}=\alpha^{2} d v+h_{a b}\left(d x^{a}+\beta^{a} d v\right)\left(d x^{b}+\beta^{b} d v\right),
$$

where $v^{\mu}$ is a Killing vector field, and hence, all the components of the metric have no dependence on the $v$-coordinate.

The norm of this vector is given by

$$
v^{\mu} v_{\mu}=g_{v v}=\alpha^{2}+\beta^{a} \beta_{a}
$$

and at the horizons is assumed to vanish. $v^{\mu}$ aligns - we assume - with a null geodesic generator $\xi^{\mu}$ so that

$$
\xi^{\nu} \xi_{; \nu}^{\mu}=\kappa \xi^{\mu},
$$

where $\kappa$ is the surface gravity along the horizon.

Now, let the horizon positions be given by constant values of a scalar function $\beta$, and let us choose $\beta^{a}$ so that it approaches smoothly to the direction given by $\partial_{\beta}$, in the vicinity of the horizons.

From this we observe that

$$
\kappa=\Gamma_{v v}^{v}=-\frac{g_{v v, \beta}}{2 s},
$$

where it is assumed that $g^{v \beta}$ goes to a non zero function $s^{-1}$ at the horizon.

Hence - by inspection of the analysis presented in section [II we should take $\frac{-1}{s} g_{v v}$ instead of $\Delta$ as a function of $\epsilon$ and $\beta$, and arrive to the conclusion that the criterion (29) must be replaced by the Cauchy-horizon-stability requirement

$$
-\left(\beta \frac{\partial^{3}}{\partial \beta^{3}}-4 \frac{\partial^{2}}{\partial \beta^{2}}\right) \frac{v^{\mu} v_{\mu}}{s} \geq 0 \text { at extreme values. }
$$

It would remain its transcription in terms of the local properties of the matter sources of the gravitational field. In view of its dependence on the first three derivatives of the metric tensor, this can be achieved using the continuity formula

$$
\frac{1}{\alpha} \partial_{v} \rho+\left.J^{b}\right|_{b}= \pm\left(S^{a b} K_{a b}+\rho K\right)+\left.\rho\right|_{b} \frac{\beta^{b}}{\alpha}-\left.2(\ln \alpha)\right|_{b} J^{b},
$$

and the Euler equation [12],

$$
\begin{aligned}
\frac{1}{\alpha} \partial_{t} J^{a}+\left.S^{a b}\right|_{b}= & \pm\left(2 J^{b} K_{b}^{a}+K J^{a}\right)+\frac{1}{\alpha} J^{a} \\
& -\left.(\ln \alpha)\right|_{b}\left(S^{a b}+\rho h^{a b}\right) .
\end{aligned}
$$


Perhaps the double-null formalism will prove to be more profitable in this goal. But the message we wanted to cross this time is merely that this can be done.

One further remark is in order. It is possible that by other means the condition $g \in C^{3}$ could be refined somewhat. However, as was pointed out in Ref. [8], the metric tensor has to be at least $C^{1}$. If this assumption is dropped, one could construct counterexamples with space-like shells of matter, where the extrinsic curvature of the shell has a jump from one side to the other; yet the weak energy condition still holds and there is safety in crossing the black-hole Cauchy horizon.

In conclusion, there is hope that the transcription of the surface-gravity inequality (10), or (34), in terms of the local properties of the matter sources of the gravitational field, would provide in more complicated situations with a reasonable set of conditions from which one may elucidate a theorem for strong cosmic censorship.

\section{Acknowledgments}

I am particularly grateful to Professor I.G Moss for various important remarks and discussions. This research has been sponsored by CONACYT of México under the grant-116020.
[1] S.W. Hawking and R. Penrose, Proc. Roy. Soc. Lond., A314, 529 (1970).

[2] R. Penrose, in General Relativity, an Einstein Centenary Survey, eds. S.W. Hawking and W. Israel, (Cambridge University Press, London, 1979); M. Simpson and R. Penrose, Internal instability in a Reissner-Nordström black hole, Int. J. Theor. Phys., 7, 183-97 (1973).

[3] J. Isenberg, V. Moncrief, Ann. of Physics 199, 84-122 (1990).

[4] S. Chandrasekhar and J.B. Hartle, Proc. Roy. Soc. (London) 484301 (1982) ; S. Chandrasekhar, The Mathematical Theory of Black Holes (Oxford University Press, New York, 1998).

[5] P. R. Brady, I.G. Moss and R. Myers, Phys. Rev. Lett. 803432 (1998).

[6] D.A. Rashdeed, Non-linear Electrodynamics: Zeroth and First Law of Black Holes Mechanics (1997); hep-th/9702087
[7] V. Husian, Phys. Rev. D 53 (4), 1759-1762 (1995); M. Visser, Lorentzian Wormholes: from Einstein to Hawking. (Springer-Verlag, New York, 1996).

[8] W. Santiago-Germán, PhD Thesis. Space-time Structure and Hidden Dimensions: University of Newcastle upon Tyne (2003); W. Santiago-Germán and I. G. Moss, Class. Quantum Grav. 18 5097-5101, (2001).

[9] J. Bardeen, presented at GR5. Tiflis, U.S.S.R., and published in the conference proceedings in the U.S.S.R (1968); E. Ayón-Beato and A. García, Phys.Lett. B 493 149-152 (2000).

[10] M. Born and L. Infeld, Proc. Roy. Soc. (London) A144, 425 (1934).

[11] G.W. Gibbons and D.A. Rasheed, Nuclear Physics B 454, 185-206 (1995),.

[12] J.W. York The Initial Value Problem and Dynamics, Gravitational Radiation, (North Holland, 1982). 